\title{
On the influence of strain on the band gap of $\mathrm{Cu}_{2} \mathrm{O}$
}

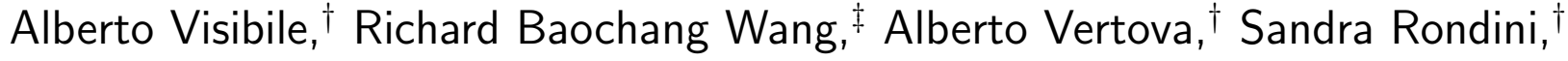 \\ Alessandro Minguzzi, ${ }^{\dagger}$ Elisabet Ahlberg, $₫$ and Michael Busch*, \\ †Universitá degli Studi di Milano, Via Golgi 1920133 Milano, Italy \\ $\ddagger$ Chalmers University of Technology, Department of Physics, SE-41296 Gothenburg, \\ Sweden \\ IDepartment of Chemistry and Molecular Biology, University of Gothenburg, Kemigården \\ 4, SE-41296 Gothenburg, Sweden \\ E-mail: michael.busch@chem.gu.se
}




\begin{abstract}
$\mathrm{Cu}_{2} \mathrm{O}$ has been considered as candidate material for transparent conducting oxides and photocatalytic water splitting. Both applications require suitably tuned band gaps. Here we explore the influence of compressive and tensile strain on the band gap by means of density functional theory (DFT) modeling. Our results indicate, that the band gap decreases under tensile strain while it increases to a maximum under moderate compressive strain and decreases again under extreme compressive strain. This peculiar behavior is rationalized through a detailed analysis of the electronic structure by means of density of states (DOS), density overlap region indicators (DORI) and crystal overlap Hamilton populations (COHP). In contrary to previous studies we do not find any indications that the band gap is determined by $\mathrm{d} 10-\mathrm{d} 10$ interactions. Instead, our analysis clearly shows that both the conduction and valence band edges are determined by $\mathrm{Cu}-\mathrm{O}$ antibonding states. The band gap decrease under extreme compressive strain is associated with the appearance of $\mathrm{Cu} 4$ sp states in the conduction band region.
\end{abstract}




\section{Introduction}

Storage of excess energy from renewable sources is a central requirement to facilitate the transformation to a sustainable and $\mathrm{CO}_{2}$ neutral society. ${ }^{1,2}$ A promising possibility for energy storage is the photoelectrochemical splitting of water into $\mathrm{H}_{2}$ and $\mathrm{O}_{2}$. One of the first discovered semiconductors for this purpose and nowadays one of the most studied materials is $\mathrm{Cu}_{2} \mathrm{O}{ }^{3,4}$ It can posses p-type semiconducting properties together with a direct band gap of $2.17 \mathrm{eV} \cdot{ }^{5}$ Furthermore it is easy to prepare through a multitude of different techniques ranging from thermal oxidation ${ }^{6}$ to electrochemical deposition ${ }^{7-13}$ and sol-gel techniques ${ }^{14-16}$ and displays suitably placed conduction band (CB) and valence band (VB) edges. ${ }^{17}$ This renders $\mathrm{Cu}_{2} \mathrm{O}$ a promising choice as photocathode. ${ }^{4}$ Besides its use in photoelectrochemical water splitting, $\mathrm{Cu}_{2} \mathrm{O}$ has also been considered as candidate for other applications such as in solar cells, ${ }^{18}$ transparent conductive oxides ${ }^{18-20}$ or optoelectronics. ${ }^{18}$

All these applications have the requirement of suitable band gaps in common. For example, in the case of transparent conducting oxides a large band gap is required to avoid absorption of visible light. ${ }^{18,19}$ For photoelectrochemical water splitting on the other hand, the band gap should be close to $1.6 \mathrm{eV}$ to maximize the use of the solar spectrum. ${ }^{21,22} \mathrm{~A}$ possibility to tune the band gap in oxides is the addition of dopants. ${ }^{21,23}$ Upon doping, the band gap is affected by two parameters, the dopant $\operatorname{size}^{24}$ and the interactions between the electronic structure of the dopant and $\mathrm{Cu}_{2} \mathrm{O} \cdot{ }^{25,26}$ Differences in the ion size are thought to introduce structural modifications in the $\mathrm{Cu}_{2} \mathrm{O}$ network which in turn modifies the $\mathrm{Cu}-\mathrm{Cu}$ interactions. ${ }^{27}$ These interactions have been reported to dominate the CB or VB. ${ }^{24,25}$ In the case of a dopant smaller than $\mathrm{Cu}^{+}$, the band gap is narrowed while it becomes wider upon addition of larger ions. ${ }^{24,25}$ Interactions between the electronic structure of $\mathrm{Cu}_{2} \mathrm{O}$ and the dopant on the other hand can result in the appearance of interband states ${ }^{28-31}$ or in the case of inert ions in the disturbance of the $\mathrm{Cu}-\mathrm{Cu}$ interactions. ${ }^{25}$

Similar changes in the band gap have also been obtained indirectly by placing $\mathrm{Cu}_{2} \mathrm{O}$ on a support material which can introduce a 2-dimensional (2D) strain through a lattice 
mismatch. This effect has been observed for $\mathrm{Cu}_{2} \mathrm{O}$ placed onto a gold support. ${ }^{32,33}$ However, under these circumstances the situation is no longer that clear since other effects such as the composition of the interface between the support and the $\mathrm{Cu}_{2} \mathrm{O}$ film or the generation of defects could also affect the band gap. ${ }^{11,12,20}$ Similarly also $\mathrm{Cu}_{2} \mathrm{O}$ placed onto a graphene oxide $^{34}$ has been studied. Both supports have a significant influence on the performance of the $\mathrm{Cu}_{2} \mathrm{O}$ photocatalyst. To which extent these differences are due to the presence of a lattice mismatch which induces a strain into $\mathrm{Cu}_{2} \mathrm{O}$ or electronic effects is still unknown. Band gap engineering has also been investigated for other systems such as graphene ${ }^{35,36}{ }^{2}$ $\mathrm{SnO}_{2}{ }^{37,38} \mathrm{FeS}_{2}{ }^{39}$ and poly(phenyl)germanes ${ }^{40}$.

In what follows we will evaluate the influence of the lattice mismatch and the influence of differences in the ion size between dopants and $\mathrm{Cu}^{+}$on the electronic structure of $\mathrm{Cu}_{2} \mathrm{O}$ by means of density functional theory (DFT) calculations. In order to isolate the changes associated with strain and remove any electronic effects, the geometric effects are mimicked by introducing 2D and 3D lattice strain. Our calculations indicate, that the presence of strain greatly affects the band gap. Interestingly, both tensile and compressive strain can result in a reduction of the band gap. This finding is taken as a basis to explore the mechanism behind the strain induced changes in the band gap of $\mathrm{Cu}_{2} \mathrm{O}$.

\section{Computational Details}

All calculations were performed with the Vienna Ab-initio Simulation Package (VASP version 5.4.1). Structures were converged using the generalized gradient approximation (GGA) $\mathrm{PBE}^{41}$ functional in combination with a plane wave cut-off of $450 \mathrm{eV}$ and a smearing of $0.1 \mathrm{eV}$. Structural convergence was assumed when the forces on all atoms were below $0.05 \mathrm{eV} / \AA$. Band gaps, density of states (DOS) and density overlap region indicators (DORI $)^{42}$ were obtained from single-point calculations at the converged GGA-PBE structures with the HSE06 ${ }^{43,44}$ range-separated hybrid functional. Valence electrons were treated 
explicitly while core-electrons were approximated within the projector augmented wavefunctions (PAW) method. ${ }^{45}$ The explicitly modeled valence shell consisted of the $2 \mathrm{~s}^{2} 2 \mathrm{p}^{4}$ electrons for $\mathrm{O}$ and of the $4 \mathrm{~s}^{1} 3 \mathrm{~d}^{10}$ electrons for $\mathrm{Cu}$.

All PBE and HSE06 calculations were performed using the minimal $\mathrm{Cu}_{2} \mathrm{O}$ unit cell consisting of $4 \mathrm{Cu}$ and $2 \mathrm{O}$ atoms with a $5 \times 5 \times 5 \mathrm{k}$-point set. The obtained lattice parameters $(\mathrm{a}=\mathrm{b}=\mathrm{c}=4.296 \AA)$ are in excellent agreement with experiment. ${ }^{46}$ Strain was induced by extending or compressing the unit cell by up to $\pm 10 \%$. Experimentally it is easy to obtain strain of at least $3 \% 47$. However, also more extreme values were included to test the hypothesis. Both 3D strain, where lattice parameters were varied along the $\mathrm{x}, \mathrm{y}$ and $\mathrm{z}$ axis and 2D strain, where the lattice parameters were only varied within the xy-plane, were considered. The relative positions of the $\mathrm{CB}$ and $\mathrm{VB}$ edges were extracted from the eigenvalues.

\section{Results and Discussion}

Assuming unstrained $\mathrm{Cu}_{2} \mathrm{O}$, our calculations indicate a direct band gap of $1.95 \mathrm{eV}$, which is in fair agreement with experiment. ${ }^{5}$ In order to evaluate the influence of strain on the band gap, two situations are considered, 3D strain along the $\mathrm{x}, \mathrm{y}$ and z-axis and 2D strain along the xy-plane. The former can be achieved through doping with ions of different size ${ }^{24}$ while the latter can be introduced through a lattice mismatch between $\mathrm{Cu}_{2} \mathrm{O}$ and a support. ${ }^{32,33,47}$ Our computations indicate a uniform decrease of the band gap under 3D tensile strain from $1.95 \mathrm{eV}$ to only $1.36 \mathrm{eV}$ (Figure 1). Upon compressing the oxide, the band gap increases from $1.95 \mathrm{eV}$ to a maximum of $2.22 \mathrm{eV}$ at $-3 \%$ compressive strain. When compressing the oxide further the band gap again decreases to $1.14 \mathrm{eV}$ at $-10 \%$ strain. An equivalent behavior has already been reported earlier for $\mathrm{Cu}_{2} \mathrm{O}$ doped with alkaline earth metal ions ${ }^{48}$ and for other materials such as $\mathrm{FeS}_{2}{ }^{39}$ and poly(phenyl)germanes. ${ }^{40}$ Identical trends are also observed when applying 2D strain (Figure 1).

In order to understand the anomalous behavior of the band gap under compressive strain 


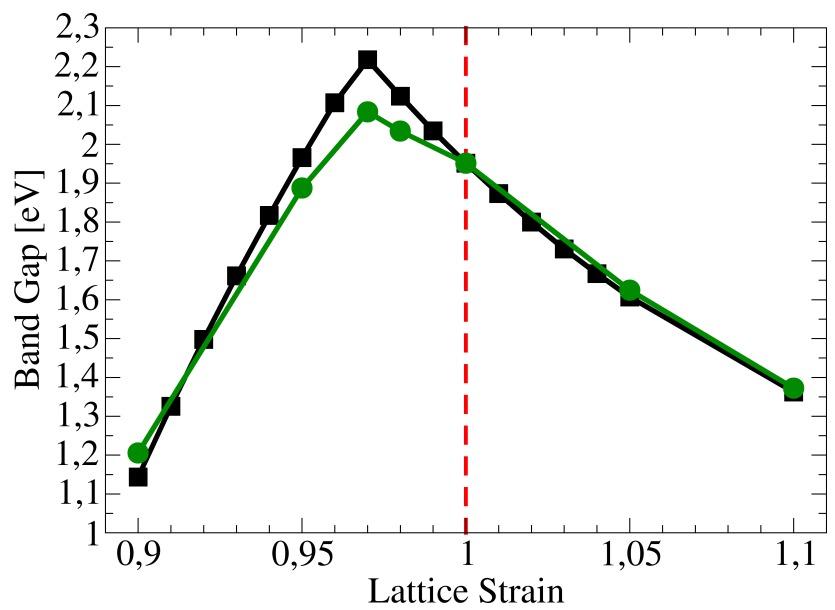

Figure 1: Summary of the influence of 2D (green circles) and 3D (black squares) strain on the band gap.

we decided to extract the positions of the CB and VB edges by using the 1s core states as reference. These states are placed within the pseudopotential and are therefore not affected by the external strain. In Figure 2, the relative positions of the CB and VB are summarized. Considering the VB, we find a monotonousmonotonic, almost linear decrease of the relative band edge energy. Similarly also the relative CB edge position decreases with the decrease of compressive strain or under tensile strain. However, the changes are no longer described by a single slope. Assuming a compressive strain larger than $-3 \%$, the CB edge energy increases linearly but is significantly less affected by the compressive strain than the VB. Accordingly, the band gap decreases when compressing $\mathrm{Cu}_{2} \mathrm{O}$. A second slope appears under less compressive or even tensile strain. In this regime, the CB edge is, similar to the VB, strongly affected. Both slopes meet at $-3 \%$ compressive strain which also corresponds to the point with the highest band gap. Due to net differences in the reaction to the strain again a decrease of the band gap is observed with increased tensile strain. The presence of two very different regimes in the $\mathrm{CB}$ may be interpreted as a first indication that different bands determine the $\mathrm{CB}$ in the two regions.

In order to obtain additional insights on the effect of strain on the CB and VB, we decided to perform a detailed analysis of the chemical bonding. Generally it is thought, that the band gap in $\mathrm{Cu}_{2} \mathrm{O}$ is determined by weak $\mathrm{d} 10-\mathrm{d} 10$ interactions between $\mathrm{Cu}$ ions. ${ }^{24,25,27,49}$ 


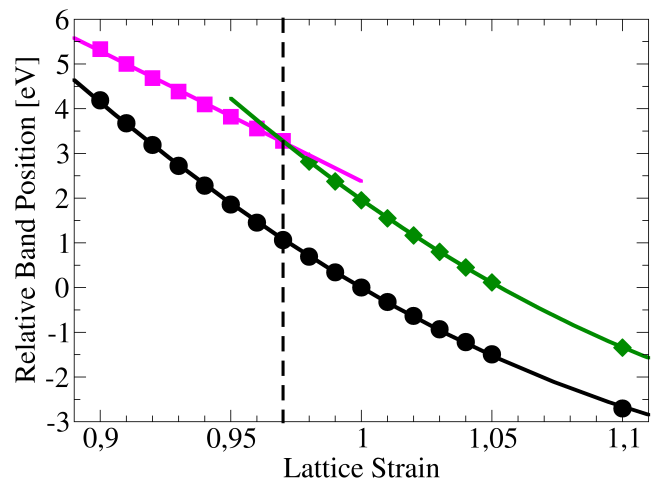

Figure 2: Relative position of the CB (magenta squares and green diamonds) and VB (black circles) edges with respect to the $\mathrm{VB}$ position in unstrained $\mathrm{Cu}_{2} \mathrm{O}$. Linear or quadratic fits have been added as guide for the eye.

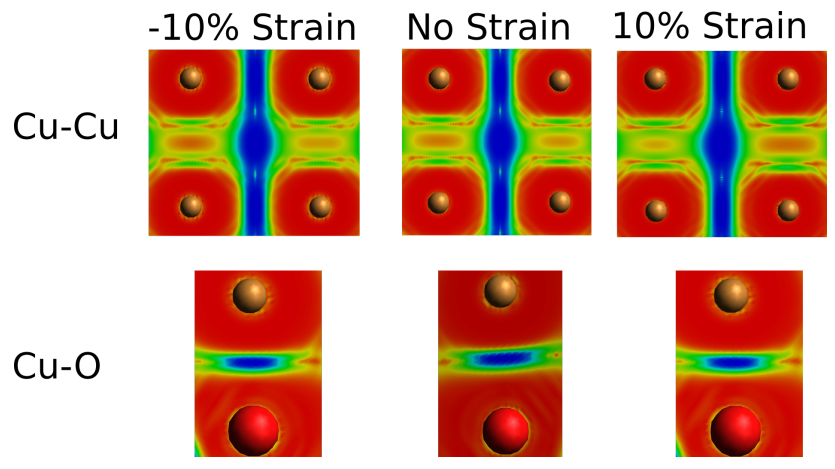

Figure 3: Density overlap region indicator (DORI) analysis of the $\mathrm{Cu}-\mathrm{O}$ and $\mathrm{Cu}-\mathrm{Cu}$ bond. The scale ranges from 0 (red) to 1 (blue). Color-code: Red - O; brown - Cu.

A possibility to qualitatively evaluate the existence and changes in the $\mathrm{Cu}-\mathrm{Cu}$ metal bond offers the density overlap region indicator (DORI). ${ }^{42}$ Mathematically, DORI corresponds to the self-referenced Laplacian of the local wave vector. ${ }^{42}$ Thus, it does not contain any information regarding the strength of the interactions but the shape of the basin (blue regions in Figure 3) between the atoms contains information regarding the presence and type of chemical bonds. ${ }^{42,50}$ Originally DORI was developed to distinguish covalent and non-covalent van-der-Waals interactions in organic molecules but recently it was shown that this tool can also be used to distinguish covalent from metallic or ionic bonds in solids. ${ }^{50}$ The DORI obtained for the $\mathrm{Cu}-\mathrm{O}$ bond is shown in Figure 3. Independent of the presence of strain three qualitatively identical plots are obtained. In all cases a localized blue basin is 
a)

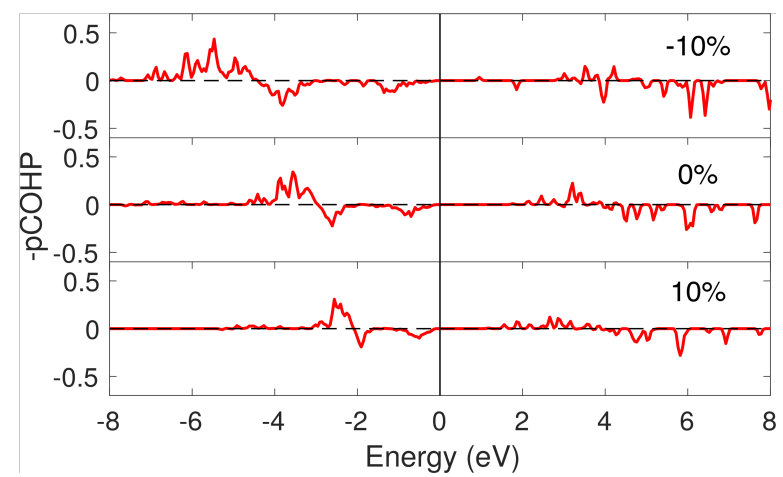

b)

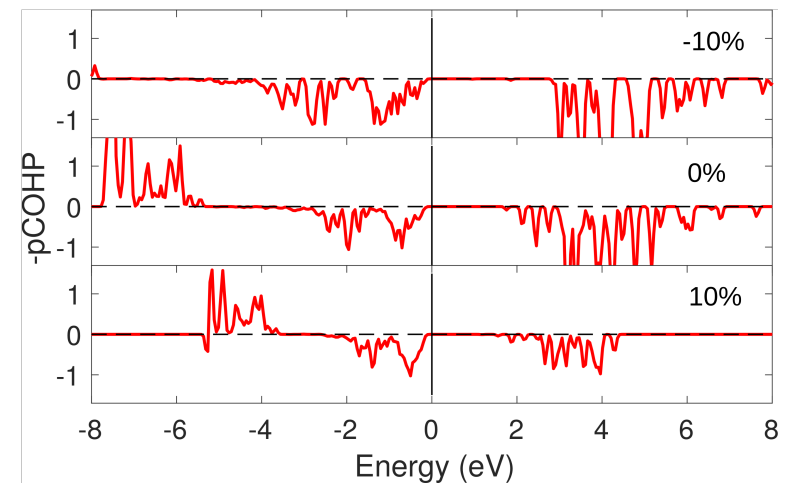

Figure 4: The crystal overlap Hamilton population (COHP) of the $\mathrm{Cu}-\mathrm{Cu}$ (a) and $\mathrm{Cu}-\mathrm{O}$ (b) bonds are depicted.

observed between $\mathrm{Cu}$ and $\mathrm{O}$. This is a typical sign of a purely covalent bond. ${ }^{42,50}$ The high degree of covalency is in line with previous work. ${ }^{25,51}$ Interestingly, no corresponding signs of covalent interactions are observed between the adjacent $\mathrm{Cu}$ ions (Figure 3). Thus, DORI does, in disagreement with previous work, ${ }^{24,25,27,49}$ not support the existence and importance of $\mathrm{d} 10-\mathrm{d} 10$ interactions between adjacent $\mathrm{Cu}$ ions in $\mathrm{Cu}_{2} \mathrm{O}$.

Nevertheless it must be kept in mind that DORI is only a qualitative tool that has not been used extensively to interpret chemical bonding in solids. Thus, the lack of $\mathrm{Cu}-\mathrm{Cu}$ interactions in DORI can only be considered as a first indication and further proof from the analysis of the partial density of states (PDOS) and crystal overlap Hamilton projector (COHP) is required. Buljan et al. ${ }^{25}$ suggested, that $\mathrm{d} 10-\mathrm{d} 10$ interactions occur between $\mathrm{Cu} \mathrm{d}_{\mathrm{z}^{2}}$ sp orbitals which determine the CB and VB. These interactions should be visible in the COHP analysis of the $\mathrm{Cu}-\mathrm{Cu}$ bond displayed in Figure 4a. In line with the DORI analysis we only observe relatively weak $\mathrm{Cu}-\mathrm{Cu}$ interactions far away from the Fermi level. 

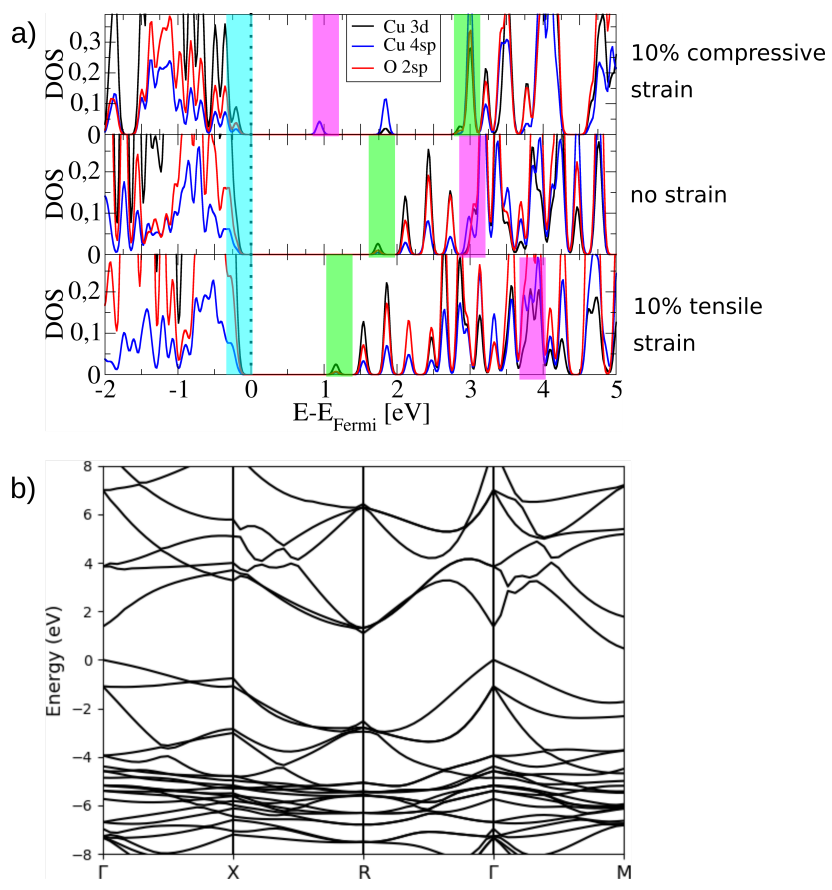

Figure 5: The projected density of states (PDOS) of pristine $\mathrm{Cu}_{2} \mathrm{O}$ and under $\pm 10 \%$ strain (a) and the band structure of $\mathrm{Cu}_{2} \mathrm{O}$ under $-10 \%$ compressive strain (b) are depicted. $\sigma^{*}$ antibonding states with relevance to the band gap width in the VB and CB regions are marked in blue and green, respectively. $\mathrm{Cu}$ 4sp states forming the $\mathrm{CB}$ under extreme compressive strain are marked in magenta.

Thus, it appears unlikely that the position of the CB and VB edges is determined by a $\mathrm{Cu}-\mathrm{Cu}$ bond. On the other hand, clear signs of antibonding $\mathrm{Cu}-\mathrm{O}$ states are observed in the $\mathrm{COHP}$ analysis (see Figure $4 \mathrm{~b}$ ). The absence of a direct $\mathrm{Cu}-\mathrm{Cu}$ bond is also in line with prior studies on $\mathrm{Cu}\left(\right.$ Phenyl) and $\mathrm{Cu}(\mathrm{Alkyl})$ tetramer and pentamer complexes ${ }^{52}$ which are isolobal to the $\mathrm{Cu}-\mathrm{O}-\mathrm{Cu}$ substructure. ${ }^{\mathrm{a}}$ These complexes display only indirect interactions between the $\mathrm{Cu}$ ions through 2 electron 3 center bonds between $\mathrm{Cu}-\mathrm{C}-\mathrm{Cu}$ fragments. ${ }^{52}$ Equivalent interactions can also be expected in $\mathrm{Cu}_{2} \mathrm{O}$ between $\mathrm{Cu}-\mathrm{O}-\mathrm{Cu}$ units.

This is further supported by our PDOS analysis which shows clear signs of strong covalent interactions between $\mathrm{Cu} 3 \mathrm{~d}$ and $4 \mathrm{sp}$ and $\mathrm{O} 2 \mathrm{sp}^{3}$ states in the $\mathrm{VB}$ and $\mathrm{CB}$ regions (green and blue regions in Figure 5a). Considering also the COHP analysis (see Figure 4), the VB is clearly determined by three degenerate $\mathrm{Cu} \mathrm{d} \mathrm{z}^{2}$ sp states forming a purely antibonding $\sigma^{*}$

\footnotetext{
${ }^{a}$ According to the isolobal principle equivalent chemical bonding between fragments must be expected if the geometry, energy and symmetry of the frontier orbitals and the number of valence electrons is equivalent. A detailed description of the isolobal principle can be found in references ${ }^{53}$ and $^{52}$
} 
bond with oxygen $\mathrm{sp}^{3}$ hybrid orbitals. A similar fully antibonding situation between $\mathrm{Cu}$ $\mathrm{d}_{\mathrm{z}^{2}}$ sp and $\mathrm{O} \mathrm{sp}^{3}$ states is also observed for the CB. Both the VB and CB are fully delocalized over the crystal structure which indicates, in agreement with the metal organic complexes, ${ }^{52}$ the presence of a delocalized multicenter bond. Pure $\mathrm{Cu} 4$ sp states, which could be involved in $\mathrm{d} 10$ - $\mathrm{d} 10$ interactions or $\mathrm{Cu}-\mathrm{Cu}$ metal bonds, are only found far away from the Fermi level (see violet region in Figure 5a). Thus, it is unlikely that $\mathrm{Cu}-\mathrm{Cu}$ interactions have a significant influence on the band gap in unstrained $\mathrm{Cu}_{2} \mathrm{O}$.

Upon applying $10 \%$ tensile strain, a significant decrease of the band gap is observed. This coincides with a shift of the bonding $\mathrm{Cu}-\mathrm{Cu}$ interactions closer to the Fermi level (see Figure 4a). Despite this shift, these states are still too far below the VB to contribute to the changes in the band gap. Additionally, the $\mathrm{Cu}-\mathrm{O} \sigma^{*}$ antibonding $\mathrm{Cu} 3 \mathrm{~d}$ and $4 \mathrm{sp}$ and $\mathrm{O}$ $\mathrm{sp}^{3}$ states forming the $\mathrm{CB}$ are shifted closer to the Fermi level which results in the observed decrease of the band gap. Under $-10 \%$ compressive strain on the other hand, the $\mathrm{Cu}-\mathrm{O}$ antibonding orbitals are destabilized (see green region in Figure 5a). Thus, an increased band gap could be expected. However, the $\mathrm{Cu} 4 \mathrm{sp}$ states, which previously were far above the Fermi level (see violet region in Figure 5a) are now shifted close to the Fermi level and form the new $\mathrm{CB}$. Additionally a second new state at $1.8 \mathrm{eV}$ consisting of $\mathrm{Cu} 3 \mathrm{~d}$ and $\mathrm{Cu}$ 4sp states is observed. These states are fully delocalized over the $\mathrm{Cu}_{2} \mathrm{O}$ crystal as indicated by the significant dispersion of the $\mathrm{CB}$ in the band structure computed at $-10 \%$ strain (see Figure 5b). Thus, they may be interpreted as an unoccupied contribution to a $\mathrm{Cu}-\mathrm{Cu}$ metal bond formed between $\mathrm{Cu} 4 \mathrm{sp}$ states. Naturally, a weak $\mathrm{Cu}-\mathrm{Cu}$ bond formed between highly dispersed $\mathrm{Cu} 4 \mathrm{sp}$ state is significantly less affected by compressive strain compared to the $\mathrm{Cu}-\mathrm{O}$ bond. This results in the significantly lower slope of the $\mathrm{CB}$ observed in Figure 2 which in turn results in a decrease of the band gap. 


\section{Conclusions}

In line with previous work, ${ }^{48}$ we have shown that $\mathrm{Cu}_{2} \mathrm{O}$ is strongly affected by external strain. Our calculations indicate, that the band gap decreases under tensile strain while it increases under weak compressive strain. Beyond a compressive strain of $-3 \%$, the band gap decreases again. The detailed analysis of the bonding situation indicates that, the turning point under compressive strain can be associated with a change of the CB. In contrast to earlier work, ${ }^{25}$ we do not find any indications for d10 - d10 interaction close to the Fermi level. Instead, the CB and VB are, under weak compressive or tensile strain, determined by $\mathrm{Cu}-\mathrm{O}$ antibonding states. Under extreme compressive strain delocalized $\mathrm{Cu} 4$ sp states which form $\mathrm{Cu}-\mathrm{Cu}$ metal bonds, are shifted close to the Fermi level and determine the CB.

\section{Acknowledgements}

Calculations were performed at C3SE through a SNIC grant. A.Visibile is grateful to Universitá degli Studi di Milano for his Ph.D. fellowship. A.M. is thankful to Universitá degli Studi di Milano for funding through the Piano Sostegno alla Ricerca 2018.

\section{References}

(1) Yang, Z.; Zhang, J.; Kintner-Meyer, M.; Lu, X.; Choi, D.; Lemmon, J.; Liu, J. Electrochemical Energy Storage for Green Grid. Chem. Rev. 2011, 111, 3577-3613.

(2) Liu, J. et al. Materials Science and Materials Chemistry for Large Scale Electrochemical Energy Storage: From Transportation to Electrical Grid. Adv. Funct. Mater. 2013, 23, 929-946.

(3) Hara, M.; Kondo, T.; Komoda, M.; Ikeda, S.; Kondo, J.; Domen, K.; Hara, M.; Shino- 
hara, K.; Tanaka, A. Cu2O as a photocatalyst for overall water splitting under visible light irradiation. Chem. Commun. 1998, 357-358.

(4) Paracchino, A.; Laporte, V.; Sivula, K.; Grätzel, M.; Thimsen, E. Highly active oxide photocathode for photoelectrochemical water reduction. Nat. Mater. 2011, 10, 456461.

(5) Moniz, S.; Shevlin, S.; Martin, D.; Guo, Z.; Tang, J. Visible-light driven heterojunction photocatalysts for water splitting ? a critical review. Energy Environ. Sci. 2015, 8, $731-759$.

(6) Li, Y.; Zhang, X.; Chen, H.; Li, Y. Thermal conversion synthesis of Cu2O photocathode and the promoting effects of carbon coating. Catal. Commun. 2015, 66, $1-5$.

(7) Wan, L.; Wang, Z.; Yang, Z.; Luo, W.; Li, Z.; Zou, Z. Modulation of dendrite growth of cuprous oxide by electrodeposition. J. Cryst. Growth 2010, 312, 3085 - 3090.

(8) Ng, S.; Ngan, A. Fabrication of nanometer-to-micron sized Cu2O single crystals by electrodeposition. Electrochim. Acta 2011, 56, 7686 - 7695.

(9) Hu, F.; Chan, K.; Yue, T.; Surya, C. Electrochemical synthesis of transparent nanocrystalline Cu2O films using a reverse potential waveform. Thin Solid Films 2014, 550, 17 $-21$.

(10) Golden, T.; Shumsky, M.; Zhou, Y.; Van der Werf, R.; Van Leeuwen, R.; Switzer, J. Electrochemical Deposition of Copper(I) Oxide Films. Chem. Mater. 1996, 8, 24992504.

(11) Brandt, I.; Tumelero, M.; Pelegrini, S.; Zangari, G.; Pasa, A. Electrodeposition of Cu2O: growth, properties, and applications. J. Solid State Electrochem. 2017, 21, 19992020. 
(12) Laidoudi, S.; Bioud, A.; Azizi, A.; Schmerber, G.; Bartringer, J.; Barre, S.; Dinia, A. Growth and characterization of electrodeposited Cu2O thin films. Semicond. Sci. Technol. 2013, 28, 115005.

(13) Paracchino, A.; Brauer, J.; Moser, J.; Thimsen, E.; Graetzel, M. Synthesis and Characterization of High-Photoactivity Electrodeposited Cu2O Solar Absorber by Photoelectrochemistry and Ultrafast Spectroscopy. J. Phys. Chem. C 2012, 116, 7341-7350.

(14) Lim, Y.; Chua, C.; Lee, C.; Chi, D. Sol?gel deposited Cu2O and CuO thin films for photocatalytic water splitting. Phys. Chem. Chem. Phys. 2014, 16, 25928-25934.

(15) Wei, M.; Huo, J. Preparation of $\mathrm{Cu} 2 \mathrm{O}$ nanorods by a simple solvothermal method. Mater. Chem. Phys. 2010, 121, $291-294$.

(16) Wei, M.; Lun, N.; Ma, X.; Wen, S. A simple solvothermal reduction route to copper and cuprous oxide. Mater. Lett. 2007, 61, 2147 - 2150.

(17) Chen, S.; Wang, l. Thermodynamic Oxidation and Reduction Potentials of Photocatalytic Semiconductors in Aqueous Solution. Chem. Mater. 2012, 24, 3659-3666.

(18) Chen, L. Review of preparation and optoelectronic characteristics of Cu2O-based solar cells with nanostructure. Mater. Sci. Semicond. Process. 2013, 16, 1172 - 1185, Advanced Oxides for Electronics.

(19) Nakano, Y.; Saeki, S.; Morikawa, T. Optical bandgap widening of p -type Cu2 O films by nitrogen doping. Appl. Phys. Lett. 2009, 94, 022111.

(20) Nolan, M. Defects in Cu2O, CuAlO2 and $\mathrm{SrCu} 2 \mathrm{O} 2$ transparent conducting oxides. Thin Solid Films 2008, 516, 8130 - 8135, Proceedings of the EMRS 2007 Fall Meeting Symposium H: Current trends in optical and x-ray metrology of advanced materials and devices II Warsaw, Poland. 
(21) Walter, M.; Warren, E.; McKone, J.; Boettcher, S.; Mi, Q.; Santori, E.; Lewis, N. Solar Water Splitting Cells. Chem. Rev. 2010, 110, 6446-6473.

(22) Bak, T.; Nowotny, J.; Rekas, M.; Sorrell, C. Photo-electrochemical hydrogen generation from water using solar energy. Materials-related aspects. Int. J. Hydrogen Energy 2002, $27,991-1022$.

(23) Brandt, I.; Tumelero, M.; Martins, C.; Pla Cid, C.; Faccio, R.; Pasa, A. Defects controlling electrical and optical properties of electrodeposited Bi doped Cu2O. J. Appl. Phys. 2018, 123, 161412.

(24) Nolan, M.; Elliott, S. Tuning the Transparency of Cu2O with Substitutional Cation Doping. Chem. Mater. 2008, 20, 5522-5531.

(25) Buljan, a.; Llunell, M.; Ruiz, E.; Alemany, P. Color and Conductivity in Cu2O and CuAlO2:? A Theoretical Analysis of d10...d10 Interactions in Solid-State Compounds. Chem. Mater. 2001, 13, 338-344.

(26) Isseroff, L.; Carter, E. Electronic Structure of Pure and Doped Cuprous Oxide with Copper Vacancies: Suppression of Trap States. Chem. Mater. 2013, 25, 253-265.

(27) Filipetti, A.; Fiorentini, V. Coexistence of ionic and metallic bonding in noble-metal oxides. Phys. Rev. B 2005, 72, 035128.

(28) Luque, A.; Martí, A. Increasing the Efficiency of Ideal Solar Cells by Photon Induced Transitions at Intermediate Levels. Phys. Rev. Lett. 1997, 78, 5014-5017.

(29) Luque, A.; Martinez, A.; Antoln, E.; Tablero, C. Intermediate bands versus levels in non-radiative recombination. Physica B 2006, 382, 320 - 327.

(30) Palacios, P.; Sánchez, K.; Conesa, J.; Fernández, J.; Wahnón, J. Theoretical modelling of intermediate band solar cell materials based on metal-doped chalcopyrite compounds. Thin Solid Films 2007, 515, 6280 - 6284. 
(31) López, N.; Reichertz, L.; Yu, K.; Campman, K.; Walukiewicz, W. Engineering the Electronic Band Structure for Multiband Solar Cells. Phys. Rev. Lett. 2011, 106, 028701.

(32) Kuo, C.; Hua, T.; Huang, M. Au Nanocrystal-Directed Growth of Au?Cu2O Core?Shell Heterostructures with Precise Morphological Control. J. Am. Chem. Soc. 2009, 131, $17871-17878$.

(33) Sai, C.; Ngac, A. Effect of core-shell structure on optical properties of Au-Cu2O nanoparticles. Physica B 2018, 532, 216 - 220, Special issue on Frontiers in Materials Science: Condensed Matters.

(34) Huang, Y.; Yan, C.; Guo, C.; Lu, Z.; Shi, Y.; Wang, Z. Synthesis of GO-modified $\mathrm{Cu} 2 \mathrm{O}$ nanosphere and the photocatalytic mechanism of water splitting for hydrogen production. Int. J. Hydrogen Energy 2017, 42, 4007 - 4016.

(35) Ni, Z.; Yu, T.; Lu, Y.; Wang, Y.; Feng, Y.; Shen, Z. Uniaxial Strain on Graphene: Raman Spectroscopy Study and Band-Gap Opening. ACS Nano 2008, 2, 2301-2305, PMID: 19206396.

(36) Gui, G.; Li, J.; Zhong, J. Band structure engineering of graphene by strain: Firstprinciples calculations. Phys. Rev. B 2008, 78, 075435.

(37) Mounkachi, O.; Salmani, E.; Lakhal, M.; Ez-Zahraouy, H.; Hamedoun, M.; Benaissa, M.; Kara, A.; Ennaoui, A.; Benyoussef, A. Band-gap engineering of SnO2. Sol. Energy Mater. Sol. Cells 2016, 148, 34 - 38, Solar Cells and Storage.

(38) Zhou, W.; Liu, Y.; Yang, Y.; Wu, P. Band Gap Engineering of SnO2 by Epitaxial Strain: Experimental and Theoretical Investigations. J. Phys. Chem. C 2014, 118, 6448-6453.

(39) Xiao, P.; Fan, X.; Liu, L.; Lau, W. Band gap engineering of FeS2 under biaxial strain: a first principles study. Phys. Chem. Chem. Phys. 2014, 16, 24466-24472. 
(40) Fa, W.; Zeng, X. Polygermanes: bandgap engineering via tensile strain and side-chain substitution. Chem. Commun. 2014, 50, 9126-9129.

(41) Perdew, J. P.; Burke, K.; Ernzerhof, M. Generalized gradient approximation made simple. Phys. Rev. Lett. 1996, 77, 3865-3868.

(42) de Silva, P.; Corminboeuf, C. Simultaneous Visualization of Covalent and Noncovalent Interactions Using Regions of Density Overlap. J. Chem. Theory Comput. 2014, 10, $3745-3756$.

(43) Heyd, J.; Scuseria, G.; Ernzerhof, M. Hybrid functionals based on a screened Coulomb potential. J. Chem. Phys. 2003, 118, 8207-8215.

(44) Heyd, J.; Scuseria, G. Efficient hybrid density functional calculations in solids: Assessment of the Heyd-Scuseria-Ernzerhof screened Coulomb hybrid functional. J. Chem. Phys. 2004, 121, 1187-1192.

(45) Blöchel, P. Projector augmented-wave method. Phys. Rev. B 1994, 50, 17953-17979.

(46) Hafner, S.; Nagel, S. The electric field gradient at the position of copper in $\mathrm{Cu} 2 \mathrm{O}$ and electronic charge density analysis by means of K-factors. Phys. Chem. Miner. 1983, 9, $19-22$.

(47) Borie, B.; Sparks Jr., C.; Cathcart, J. Epitaxially Induced Strains in cu2O Films on Copper Single Crystalls-I X-Ray Diffraction Effects. Acta Metallurgica 1962, 10, 691702.

(48) Nie, X.; Wei, S.; Zhang, S. First-principles study of transparent p-type conductive SrCu2O2 and related compounds. Phys. Rev. B 2002, 65, 0751111-0751118.

(49) Caballero-Briones, F.; Palacios-Padros, A.; Calzadilla, O.; Moreira, I.; Sanz, F. Disruption of the Chemical Environment and Electronic Structure in p-Type $\mathrm{Cu}_{2} \mathrm{O}$ Films by Alkaline Doping. J. Phys. Chem. C 2012, 116, 13524-13535. 
(50) Busch, M.; Wang, R.; Hellman, A.; Rossmeisl, J.; Grönbeck, H. The Influence of Inert Ions on the Reactivity of Manganese Oxides. J. Phys. Chem. C 2018, 122, 216-226.

(51) Laskowski, R.; Blaha, P.; Schwarz, K. Charge distribution and chemical bonding in Cu2O. Phys. Rev. B 2003, 67, 075102.

(52) Elschenbroich, C. Organometallics, 3rd ed.; Wiley-VCH: Weinheim, 2006.

(53) Hoffmann, R. Building Bridges Between Inorganic and Organic Chemistry (Nobel Lecture). Angew. Chem. Int. Ed. 1982, 21, 711-724. 